\title{
Rapid resolution of femoral head osteonecrosis after rotational acetabular osteotomy
}

\author{
Masahiko Nozawa $\cdot$ Keiji Matsuda $\cdot$ Katsuhiko Maezawa $\cdot$ Sungon Kim $\cdot$ \\ Kouichi Maeda - Takashi Ikegami · Reiko Kubota - Kentaro Hayashi · \\ Masataka Nagayama · Haruka Kaneko
}

Received: 8 March 2008/Accepted: 21 September 2008/Published online: 25 October 2008

(C) Springer-Verlag 2008

\begin{abstract}
The natural history of osteonecrosis of the femoral head is generally thought to be one of progressive deterioration if no intervention is undertaken. However, it is unknown whether surgical intervention is beneficial for patients with a small region of osteonecrosis. We observed rapid improvement of MRI findings after rotational acetabular osteotomy (RAO) was performed in a young patient with osteonecrosis of the femoral head. The band-like low signal area on T2-weighted images almost resolved by six months after surgery. He returned to work as an electrician by six months after surgery. Early surgical intervention such as RAO that alters the mechanical force acting on the necrotic region of the femoral head may accelerate the recovery of osteonecrosis and the improvement of symptoms.
\end{abstract}

Keywords Osteonecrosis of the femoral head .

MRI finding · Rotational acetabular osteotomy

\section{Introduction}

Joint-preserving procedures for the treatment of osteonecrosis of the femoral head in young patients include core decompression, vascularized bone grafting, and proximal femoral osteotomy. Since 1995, we have performed rotational acetabular osteotomy (RAO) for 90 hips with

M. Nozawa $(\bowtie) \cdot$ K. Matsuda $\cdot$ K. Maezawa $\cdot$ S. Kim ·

K. Maeda · T. Ikegami · R. Kubota · K. Hayashi ·

M. Nagayama $\cdot$ H. Kaneko

Department of Orthopaedic Surgery,

Juntendo University Nerima Hospital,

3-1-10 Takanodai, Nerima-ku, Tokyo 177-8521, Japan

e-mail: nozawa@juntendo-nerima.jp osteonecrosis associated with collapse of the femoral head in young patients [1]. The rationale behind the use of this procedure to treat osteonecrosis of the femoral head is that it shifts the weight-bearing region to articular cartilage that is still supported by the intact lateral cortex of the femoral head. We observed interesting MRI changes in a 39-yearold man with osteonecrosis of the femoral head at six months after RAO, which are reported here.

\section{Case report}

A 39-year-old man presented with osteonecrosis of the right hip. He complained of severe right hip pain during rest as well as on walking for one year before his first visit. There was an obvious limp when he walked. An anterior radiograph showed minor discontinuity of the bone trabeculae in the medial one-third of the weight-bearing region of the femoral head and slight collapse of the femoral head (Fig. 1). This osteonecrosis was graded as stage III-A according to the method of Steinberg et al. [2]. MRI displayed typical features of osteonecrosis of the femoral head in the same region (Fig. 2). Three-dimensional CT also showed collapse of the femoral head (Fig. 3). He underwent RAO of the right hip in June 2007 using the technique of Ninomiya and Tagawa [3]. There were no complications during surgery or the postoperative period. Use of a wheel was allowed from one week postoperatively, and non-weight-bearing walking on two crutches was commenced after the second postoperative week. Partial weight-bearing was permitted at two months after surgery, and full weight-bearing was allowed at four months.

At follow-up, the patient had no pain and could walk without crutches. He returned to work as an electrician by 
Fig. 1 a Anteroposterior radiograph shows faint bone trabeculae in the medial onethird of the weight-bearing region of the femoral head. b Lateral radiograph shows a small depression in the center of the femoral head
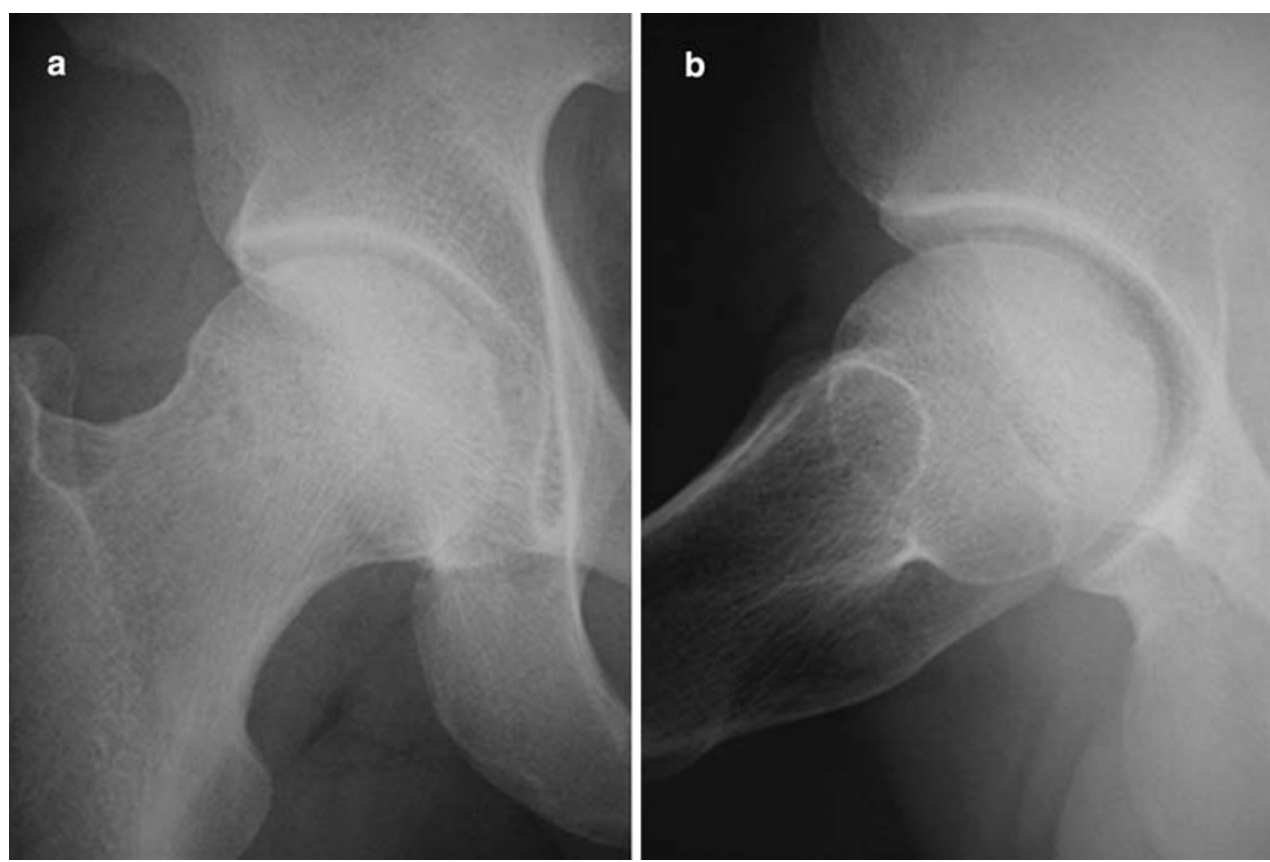

Fig. 2 a T2-weighted MR image shows a small band-like area in the midsagittal slice. $\mathbf{b}$ Fat suppression image shows a high intensity in the same region
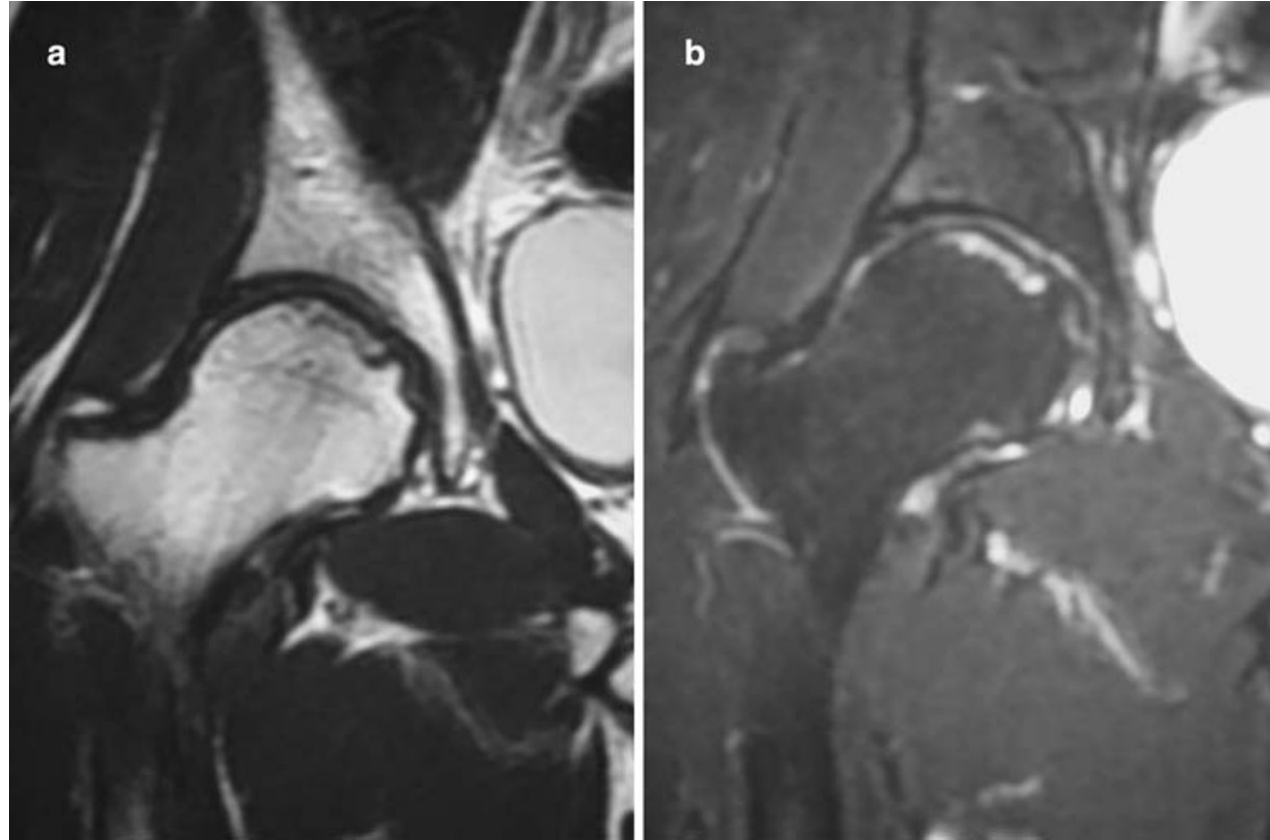

six months after surgery. An anterior radiograph showed improvement of the bony trabeculae and complete resolution of osteonecrosis was revealed by MRI at six months (Figs. 4, 5). The patient provided consent to the publication of this case report.

\section{Discussion}

The natural history of osteonecrosis of the femoral head is generally thought to be one of progressive deterioration if no intervention is undertaken. However, it is unknown whether surgical intervention is beneficial for patients with a small region of osteonecrosis. The earliest MRI finding is a low signal intensity line on T2-weighted images that represents the interface between normal and ischemic bone, and is also called a band. This change was demonstrated in our patient. There is controversy about whether or not the presence of asymptomatic disease is an indication for a joint-preserving procedure. Joint-preserving surgical intervention is generally more successful at an earlier stage of the disease than after the occurrence of subchondral 


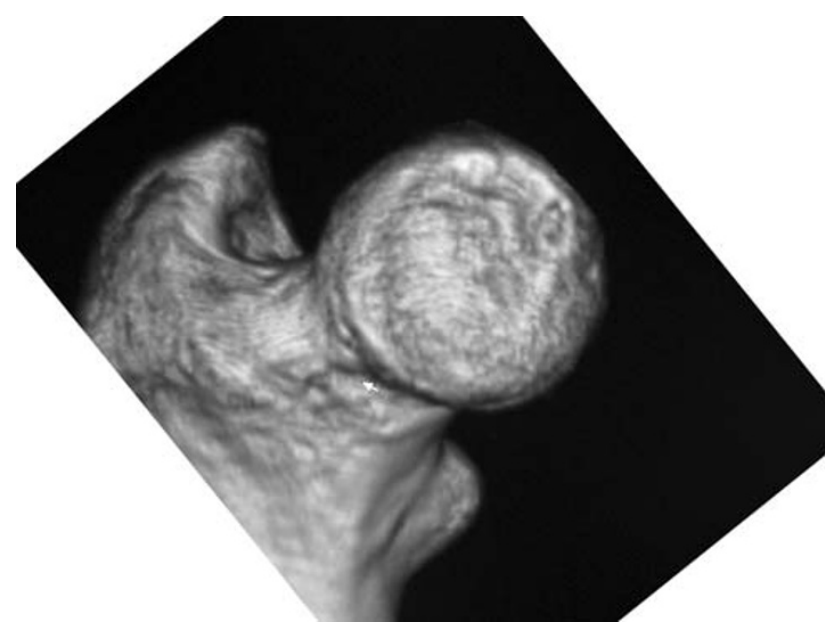

Fig. 3 3DCT shows mild collapse of the femoral head

fracture $[4,5]$. On the other hand, Cheng et al. reported three hips with evidence of spontaneous resolution on serial magnetic resonance images and stated that prolonged observation over several years is necessary in order to detect evidence of resolution with asymptomatic disease [6]. Kopecky et al. reported that in some patients with smaller AVN lesions, symptoms do not develop and the abnormalities seen on MRI occasionally improve spontaneously [7]. Mulliken et al. performed a prospective study with MRI and found that 15 hips of 132 renal transplant recipients developed osteonecrosis, with 11 of these 15 hips being initially asymptomatic and only one lesion showing progression during an average follow-up period of 22 months [8]. Ohzono et al. described that 13 out of 16 hips with osteonecrosis similar to our case in size, location of necrotic lesion and stage did not progress to collapse at an average follow-up of over five years radiographically [9]. On the other hand, Hernigou et al. reported that all of 45 symptomatic hips which were graded as stage-II or stage-III osteonecrosis according to the Steinberg classification with sickle-cell disease progressed to stage IV without surgical intervention [10]. There have been few reports about the resolution of symptomatic osteonecrosis on MRI. In our patients, resolution of osteonecrosis was complete at six months after RAO on radiographs and MRI, possibly because redistribution of weight-bearing force led to accelerated remodeling of the necrotic region. Though we have performed RAO for the treatment of osteonecrosis of the femoral head with collapse in 90 hips, we have not found early resolution of the MRI changes like that recognized in this case. Atsumi et al. [11] reported the effectiveness of posterior rotational osteotomy for nontraumatic osteonecrosis with extensive collapse in young patients, but they did not mention the changes in MRI findings after surgery. Chan et al. reported two cases in which a small region of ostenecrosis decreased in size on MRI at one year after core decompression and bone grafting [12].

There is a correlation between the outcome and the extent of osteonecrosis, whether the hip is treated conservatively or subjected to an operative procedure. We believe that achieving adequate acetabular coverage of the viable lateral portion of the femoral head by RAO was the most important factor leading to resolution of necrosis in this case.
Fig. 4 a Remodeling of bone trabeculae in the osteoncrotic region is revealed by an anteroposterior radiograph at six months after surgery. b Lateral radiograph also shows remodeling of trabeculae
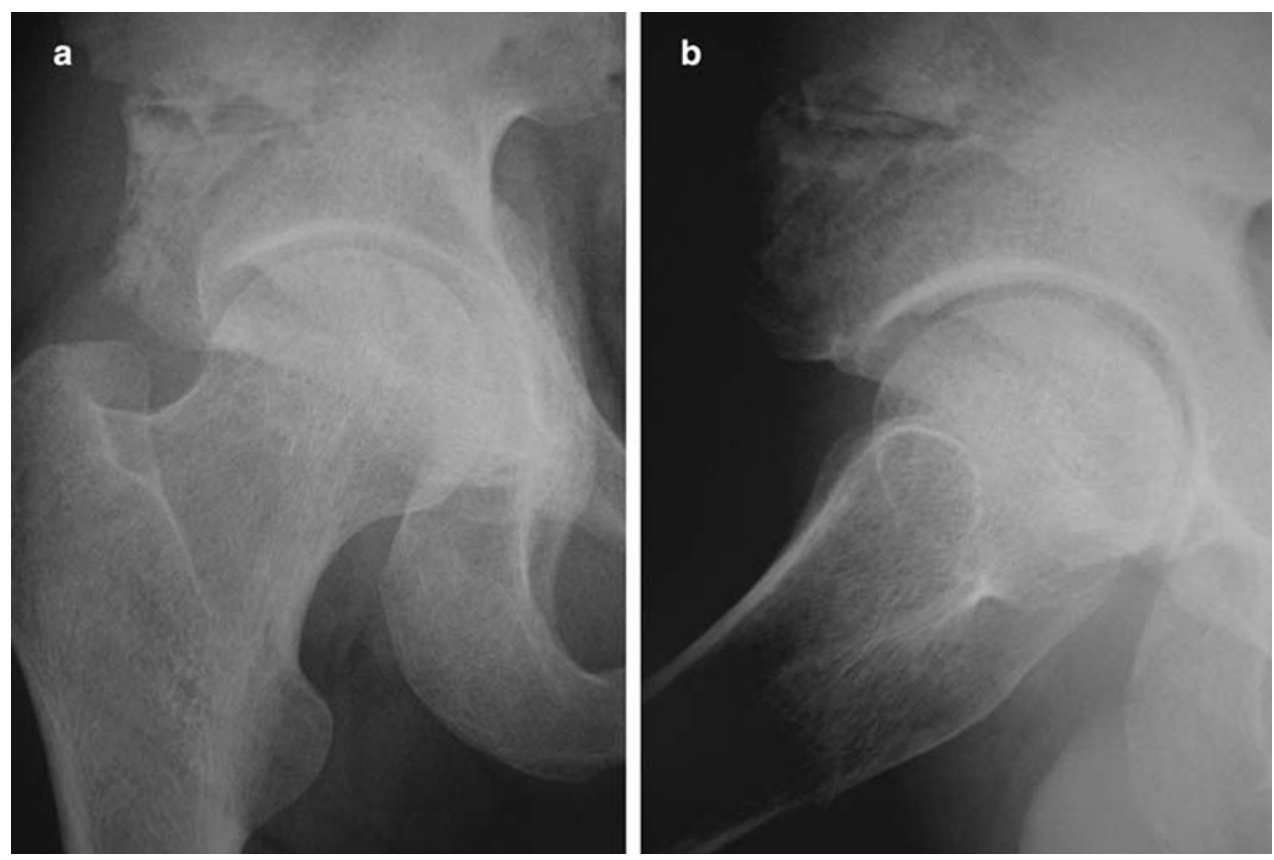
Fig. 5 a The T2-weighted MR image is almost normal. b The fat suppression image also shows almost normal findings
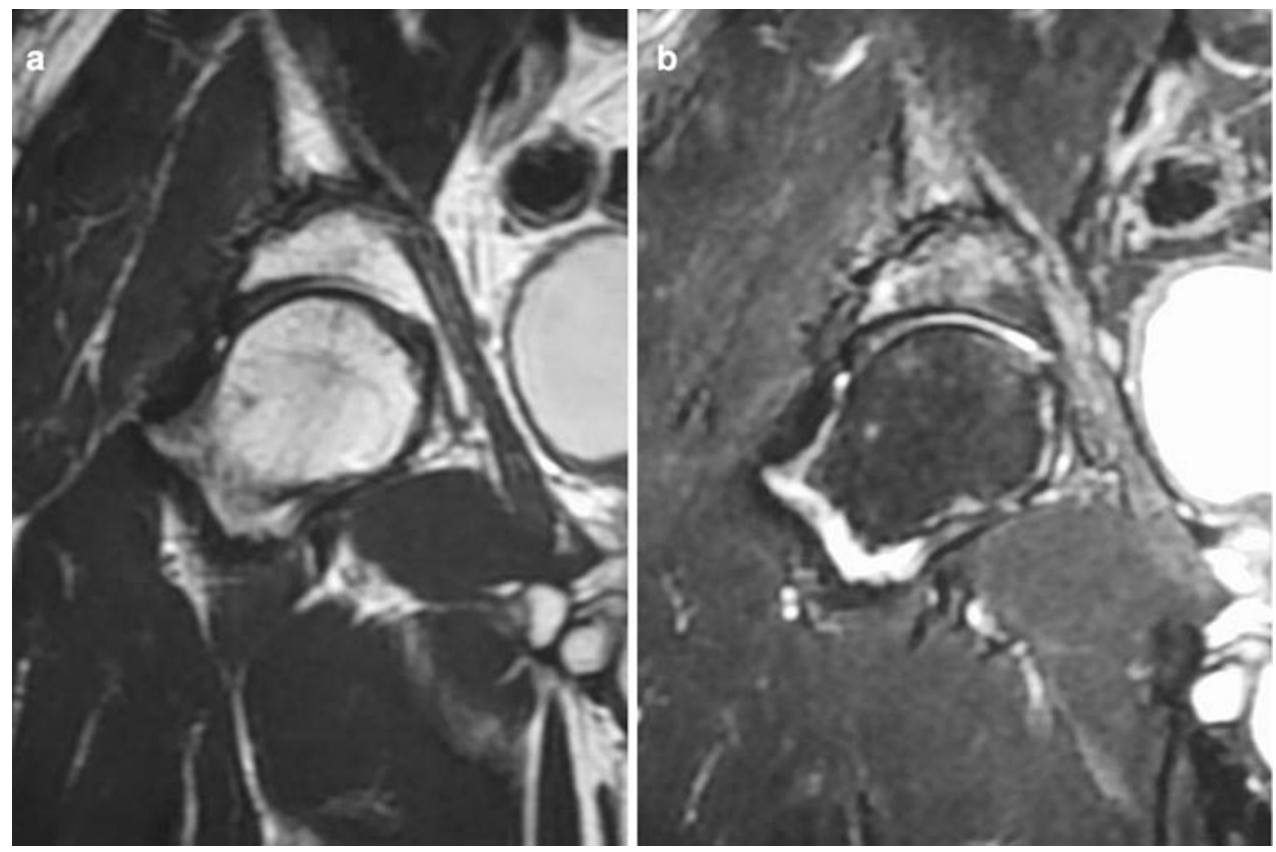

In summary, early surgical intervention such as RAO that alters the mechanical force acting on the necrotic region of the femoral head may accelerate the recovery from osteonecrosis and the improvement of symptoms.

Conflict of interest statement We disclose any financial and personal relationships with other people or organizations that could inappropriately influence their work. Examples of potential conflicts of interest include employment, consultancies, stock ownership, honoraria, paid expert testimony, patent applications/registrations, and grants or other funding.

\section{References}

1. Ninomiya S, Tagawa H (1984) Rotational acetabular osteotomy for the dysplastic hip. J Bone Joint Surg Am 66:430-436

2. Steinberg ME, Corces A, Fallon M (1999) Acetabular involvement in osteonecrosis of the femoral head. J Bone Joint Surg Am 81:60-65

3. Nozawa M, Enomoto F, Shitoto K, Matsuda K, Maezawa K, Kurosawa H (2005) Rotational acetabular osteotomy for osteonecrosis with collapse of the femoral head in young patients. J Bone Joint Surg Am 87:514-520

4. Ficat RP (1985) Idiopathic bone necrosis of the femora head. J Bone Joint Surg Br 67:3-9
5. Steinberg ME, Brighton CT, Corces A, Hayken GD, Steinberg DR, Strafford B, Tooze SE (1989) Osteonecrosis of the femoral head. Clin Orthop 249:199-209

6. Cheng EY, Thongtrangan I, Laorr A, Saleh KJ (2004) Spontaneous resolution of osteonecrosis of the femoral head. $J$ Bone Joint Surg Am 86:2594-2599

7. Kopecky KK, Braunstein EM, Brandt KD, Filo RS, Leapman SB, Capello WN, Klatte EC (1991) Apparent avascular necrosis of the hip: appearance and spontaneous resolution of MR findings in renal allograft recipients. Radiology 179:523-527

8. Mulliken BD, Renfrew DL, Brand RA, Whitten CG (1994) Prevalence of previously undetected osteonecrosis of the femoral head in renal transplant recipients. Radiology 192:831-834

9. Ohzono K, Saito M, Takaoka K, Ono K, Saito S, Nishina T, Kadowaki T (1991) Natural history of nontraumatic avascular necrosis of the femoral head. J Bone Joint Surg Br 73:68-72

10. Hernigou P, Bachir D, Galacteros F (2003) The natural history of symptomatic osteonecrosis in adults with sickle-cell disease. J Bone Joint Surg Am 85:500-504

11. Atsumi T, Kajihara T, Hiranuma Y, Tamaoki S, Asakura Y (2006) Posterior rotational osteotomy for nontraumatic osteonecrosis with extensive collapsed lesions in young patients. J Bone Joint Surg Am 88:42-47

12. Chan TW, Dalinka MK, Steinberg ME, Kressel HY (1991) MRI appearance of femoral head osteonecrosis following core decompression and bone grafting. Skeletal Radiol 20:103-107 